\title{
Gains in life expectancy after elimination of major causes of death: revised estimates taking into account the effect of competing causes
}

\author{
J P Mackenbach, A E Kunst, H Lautenbach, Y B Oei, F Bijlsma
}

Department of Public Health, Erasmus University, Rotterdam, the Netherlands

J P Mackenbach

A E Kunst

H Lautenbach

Division of Health and Social Well-being, Statistics Netherlands, Voorburg, the

Netherlands

Y B Oei

F Bijlsma

Correspondence to: Professor Dr J P

Mackenbach, Department of Public Health, Erasmus University, PO Box 1738 3000 DR Rotterdam, the Netherlands.

Accepted for publication 16 June 1998

\begin{abstract}
Background -It is generally acknowledged that conventional estimates of the potential number of life years to be gained by elimination of causes of death are too generous. This is because these estimates fail to take into account the fact that those who are saved from the cause are likely to have one or more other conditions ("competing" causes of death), which may increase their risks of dying. It is unknown to what extent this introduces bias in comparisons of life years to be gained between underlying causes of death. The purpose of the study was to assess this bias.
\end{abstract}

Data and methods-A sample of 5975 death certificates from the Netherlands, 1990, was coded for the presence of diseases that, according to a set of explicit rules, could be regarded as potential causes of death "competing" with the underlying cause. Logistic regression analysis was used to quantify age and sex adjusted differences between four main underlying causes of death (neoplasms, cardiovascular diseases, respiratory diseases, all other diseases) in prevalence of the six most frequent competing causes of death (neoplasms, ischaemic heart disease, cerebrovascular disease, other cardiovascular diseases, chronic obstructive lung disease, all other diseases). These prevalence differences were then used to revise conventional calculations of gains in life expectancy, by taking them to indicate differences in risk of dying from these competing causes after the underlying cause has been eliminated.

Results-The prevalence of competing causes of death is relatively low among persons dying from neoplasms as the underlying cause, about average among persons dying from cardiovascular diseases, and relatively high among persons dying from respiratory diseases. Taking this into account results in substantial decreases of potential life years to be gained by elimination of cardiovascular diseases and respiratory diseases, relative to the number of years to be gained by elimination of neoplasms. Specifically, while according to the conventional calculations the gain in life expectancy by elimination of cardiovascular diseases exceeds that for neoplasms by more than one year, in the revised calculations the number of life years to be gained is approximately equal.
Conclusions-Despite its limitations, mainly relating to reliance on death certificate data, this study suggests that conventional estimates of differences between underlying causes of death in life years to be gained by elimination are seriously biased by ignoring the effects of competing causes. Specifically, the relative impacts of eliminating cardiovascular diseases and respiratory diseases, as compared with eliminating neoplasms, seem to be overestimated. The implications are discussed. (F Epidemiol Community Health 1999;53:32-37)

Estimates of the number of life years to be gained by elimination of specific causes of death provide an easy to grasp and powerful summary of the relative importance of these causes of death, as well as of the potential benefits of intervention programmes. ${ }^{1-5}$

Unfortunately, however, conventionally derived estimates are likely to be biased. The main assumption on which cause-elimination life table calculations rest is, that those who are saved from dying because of the eliminated cause (for example, ischaemic heart disease) have risks of dying from non-eliminated causes that are equal to the average risks of dying from these causes as observed in the total population. ${ }^{16-8}$ This assumption is far from realistic: because of common risk factors many persons dying from ischaemic heart disease will be at a higher than average risk of dying from various other causes, such as lung cancer (smoking), cerebrovascular disease (hypertension, arteriosclerosis), and chronic obstructive lung disease (smoking). Gains in life expectancy after elimination of ischaemic heart disease are therefore likely to be too optimistic. Moreover, this element of overestimation may differ by underlying cause of death, thereby introducing bias in the comparison between the effects of eliminating different causes of death. If among patients saved from dying caused by lung cancer or breast cancer, the remnant mortality risk would be less (or more) increased than it is among patients saved from dying because of ischaemic heart disease, the difference between these causes in life years gained would be smaller (or larger).

Although this problem of "competing" causes of death, and its potential implications for cause-elimination life tables, has frequently been discussed in the epidemiologic and demographic literature, ${ }^{6-10}$ a lack of empirical data on associations between causes of death has until now prevented a solution from being 
developed. Nevertheless, a data source exists that provides opportunities for studying associations between causes of death: death certificates not only contain information on underlying causes of death, but also on other conditions present at the moment of dying. ${ }^{11}{ }^{12}$ In several countries information on other conditions mentioned on the death certificate is made available in the form of multiple cause of death statistics, ${ }^{13-16}$ but these have only rarely been used to adjust conventional causeelimination calculations, ${ }^{9}{ }^{17}$ even though according to an authoritative review this would be one of the most promising applications. ${ }^{11}$ This is perhaps because not all other conditions present at the moment of dying, and included in multiple cause of death statistics, can be regarded as "competing" causes of death, or to be more precise, conditions that in the case of elimination of the underlying cause could develop into a "new" underlying cause and eventually kill the patient. For example, some of these other conditions may actually be consequences of the condition that has been identified as the underlying cause (for example, congestive heart failure in the case of ischaemic heart disease, or pneumonia in the case of lung cancer). In the case of elimination of the underlying cause, these consequences will also be eliminated. It is therefore necessary to critically evaluate the information on death certificates, and to decide for each condition whether, given the underlying cause, it can be regarded as a competing cause.

In the study reported in this paper we have coded a sample of death certificates from the Netherlands for the presence of competing causes of death, and used this information to adjust conventional estimates of gains in life expectancy for the presence of these competing causes. The main purpose was to assess the extent to which conventional estimates of differences in life years to be gained between four main groups of underlying causes of death (neoplasms, cardiovascular diseases, respiratory diseases, and all other diseases) are biased by differences between these underlying causes in prevalence at death of competing causes.

\section{Methods}

MEASUREMENT OF ASSOCIATIONS BETWEEN CAUSES OF DEATH

Data on the prevalence of diseases present at the moment of death were obtained for a sample of 5975 certificates among deaths occurring in the Netherlands in 1990. To increase the statistical power to detect differences between underlying causes of death, this sample was stratified by four broad groups of underlying causes of death: neoplasms $(30 \%$ of the sample), cardiovascular diseases $(30 \%)$, respiratory diseases $(20 \%)$, and other diseases $(20 \%)$. Deaths resulting from ill defined or external causes of death were excluded. For each broad group of causes of death separately, the sample was in addition stratified by five year age group, with age groups being weighted according to the total number of life years that were lost in the Netherlands in 1990 because of deaths in the respective age groups.
In a special research effort, each death certificate was coded as follows. Firstly, the underlying cause of death was identified on the basis of the code as assigned by Statistics Netherlands during routine codings according to the ICD-9 rules. ${ }^{18}$ Secondly, other conditions mentioned anywhere on the certificate were coded, if they met each of the following criteria: (1)The condition is apparently not a complication or side effect of the underlying or of another identified competing cause of death. In other words, the condition originated independently from other causes of death given at the certificate. (2)The condition or its consequences has a longlasting or chronic nature. (3)The condition has the potential to develop into a new underlying cause of death in the hypothetical case that the original underlying cause would be eliminated, or it has the potential to give rise to the emergence of a disease that could develop into a new underlying cause of death.

The first and third criteria specify the main characteristics of competing causes of death: independence from the underlying cause of death and the potential to become an underlying cause of death itself. The second criterion was added to exclude acute conditions such as influenza, whose presence implies an increased death risk for a short period only. The second part of the third criterion was added to include biological risk factors for disease, such as obesity and hypertension. Although these risks factors do not, or only rarely, become an underlying cause of death, their presence implies an increased risk for the development of other diseases, which in turn could lead to death. More details of the coding procedure can be found elsewhere. ${ }^{19}$

Differences between underlying causes of death with respect to the prevalence of specific competing causes of death were expressed by means of age and sex adjusted odds ratios. These odds ratios were estimated by means of logistic regression. In the regression model, the prevalence of a specific competing cause of death was expressed as a function of sex/age group (males and females/ 0-54, 55-69, 70-79, and $80+$ years) and broad group of underlying cause of death (neoplasms, cardiovascular diseases, respiratory diseases, and other diseases).

CAUSE ELIMINATION LIFE TABLE CALCULATIONS The cause-limination life table consists of two populations: the population L, representing the living population, and the population $S$, representing those saved from dying from disease $\mathrm{A}$. Population L is constructed according to standard techniques, which require as input the probabilities of dying in the age interval $x, x+n$ $\left({ }_{n} q_{x}\right)$ that are observed for the general population. Population $S$ is constructed in two steps. Firstly, mortality quotients for cause of death A are calculated, and the observed or "dependent" cause specific mortality quotients ${ }_{n} q_{x . A}$ are converted to "net" or "independent" cause specific quotients ${ }_{n} Q_{x . A}$ by the formula of Chiang. ${ }^{1}$ Then the quotients ${ }_{n} \mathrm{Q}_{\mathrm{x} . \mathrm{A}}$ are used to calculate the number of deaths from $\mathrm{A}$ in $\mathrm{L}$, and those who die from $\mathrm{A}$ are entered into 
Table 1 Prevalence of competing causes of death, by main group of underlying causes of death. The Netherlands, sample, 1990

\begin{tabular}{|c|c|c|c|c|c|}
\hline \multirow[b]{2}{*}{ Competing cause of death ${ }^{\star}$} & \multirow{2}{*}{$\begin{array}{l}\text { Overall } \\
\text { prevalence in } \\
\text { sample } \\
(\%)\end{array}$} & \multicolumn{4}{|c|}{ Odds ratio $(95 \%$ CI) + by underlying cause of death $\neq$} \\
\hline & & Neoplasms & $\begin{array}{l}\text { Cardiovascular } \\
\text { diseases }\end{array}$ & $\begin{array}{l}\text { Respiratory } \\
\text { diseases }\end{array}$ & Other diseases \\
\hline Neoplasms & 2.04 & 1.00 & $0.77(0.48,1.23)$ & $1.15(0.71,1.85)$ & $0.86(0.47,1.56)$ \\
\hline Ischaemic heart disease & 3.33 & 1.00 & $1.31(0.83,2.07)$ & $1.56(1.04,2.32)$ & $2.23(1.47,3.38)$ \\
\hline Cerebrovascular disease & 3.09 & 1.00 & $3.62(2.19,5.95)$ & $2.64(1.56,4.48)$ & $4.07(2.38,7.03)$ \\
\hline Other cardiovascular disease & 10.72 & 1.00 & $4.98(3.66,6.76)$ & $6.38(4.69,8.66)$ & $3.32(2.35,4.68)$ \\
\hline Chronic obstructive lung disease & 2.89 & 1.00 & $1.29(0.90,1.86)$ & $0.63(0.32,1.23)$ & $1.13(0.70,1.82)$ \\
\hline All other diseases & 24.15 & 1.00 & $2.51(2.08,3.02)$ & $4.59(3.77,5.89)$ & $3.44(2.81,4.21)$ \\
\hline Number of deaths in sample & 5975 & 1797 & 1793 & 1196 & 1189 \\
\hline
\end{tabular}

*ICD-9 codes: neoplasms 140-239; ischaemic heart disease 410-414; cerebrovascular disease 430-438; other cardiovascular disease R (390-459); chronic obstructive lung disease 490-496; all other diseases R (001-799). †Adjusted for differences in age and sex distribution between underlying causes of death. $¥$ ICD-9 codes: neoplasms 140-239; cardiovascular disease 390-459; respiratory disease 460-519; all other diseases R (001-799).

population $\mathrm{S}$, which has the same mortality risks as population $\mathrm{L}$. Those in $\mathrm{S}$ who would die again from cause $A$, however, remain in $S$.

The life expectancy of population L is calculated according to standard techniques in which the total number of years lived by population $\mathrm{L}$ is divided by the size of that population at birth $\left(1_{0}\right)$. The life expectancy after elimination of cause of death $\mathrm{A}$ is calculated by adding to the numerator the total number of years lived by population $S$. The difference between the two life expectancies is equal to the gain in life expectancy by eliminating A. ${ }^{1}$

We applied this basic approach to the Netherlands for 1990. The calculations were based on data on the number of deaths by sex, one year age group $(0-98,99+)$ and cause of death. Like the cause of death data, the corresponding numbers of births by sex and numbers of population by age and sex were obtained from the continuous population registry.

The crucial assumption in the standard cause elimination life table is that the mortality quotients ${ }_{n} Q_{x}$ for the general population also apply to those saved from dying from cause A. In addition, as the same procedure is followed with respect to the elimination of whatever other disease, for example $\mathrm{B}$, the assumption is made that the population that is saved from dying from $\mathrm{B}$ has the same mortality risks as those saved from dying from A. In this paper, we will abandon the latter assumption and allow for differences between those saved from dying from $\mathrm{A}$ and those saved from dying from $\mathrm{B}$ in the risk of dying from third diseases, say $\mathrm{C}$.

We will do this on the basis of the following formula:

$$
\frac{{ }_{n} Q_{x \cdot C(-A)}}{{ }_{n} Q_{x \cdot C(-B)}}=\frac{P_{C(A)} /\left(1-P_{C(A)}\right)}{P_{C(B)} /\left(1-P_{C(B)}\right)}
$$

in which

${ }_{n} Q_{x . C(-A)}$ (independent) probability of dying from $\mathrm{C}$ among those saved from dying from $\mathrm{A}$

${ }_{n} Q_{x . C(-B)}$ (independent) probability of dying from C among those saved from dying from $B$
$\mathrm{P}_{\mathrm{C}(\mathrm{A})} /\left(1-\mathrm{P}_{\mathrm{C}(\mathrm{A})}\right)$ prevalence odds of $\mathrm{C}$ among those dying from $\mathrm{A}$

$\mathrm{P}_{\mathrm{C}(\mathrm{B})} /\left(1-\mathrm{P}_{\mathrm{C}(\mathrm{B})}\right) \quad$ prevalence odds of $\mathrm{C}$ among those dying from $B$

The main assumption underlying this approach is, that the prevalence odds ratios for $\mathrm{C}$ as obtained in the regression analyses (see above) can be used as a proxy of the mortality risk ratios for $\mathrm{C}$. This is likely to be valid, as long as the case fatality of $\mathrm{C}$ will not differ between patients dying (saved) from $\mathrm{A}$ and those dying (saved) from $\mathrm{B}$. The (average) risks of dying from $\mathrm{C}$ (that is, all separate competing causes) were taken from Dutch cause of death statistics.

The cause elimination calculation will be carried out for four main groups of underlying causes of death (neoplasms, cardiovascular diseases, respiratory diseases and all other diseases). In the case of elimination of such a broad group, only competing causes that are in another broad group will be assumed to remain; competing causes that are part of the same broad group will be eliminated with the underlying cause.

\section{Results}

In the sample of 5975 death certificates, 2762 competing causes were identified. This corresponds to 46.2 competing causes per 100 deaths. Only five categories of competing causes were found in more than $1 \%$ of all deaths (neoplasms, ischaemic heart disease, cerebrovascular disease, other cardiovascular disease, chronic obstructive lung disease) and these were used for further analyses, together with a category of all other diseases. The age and sex adjusted prevalence of some of these competing causes differed substantially by underlying cause group. Because this prevalence usually was lowest among deaths from neoplasms as the underlying cause, this was used as the reference category for the logistic regression analysis (table 1 ). There is no clear variation between underlying causes of death in the prevalence of either neoplasms or chronic obstructive lung disease as competing causes of death. Note that in the case of neoplasms as the underlying cause, neoplasms as a competing cause only includes second cancers (origina- 
ting from a different tissue and/or organ) in the same patient. The prevalence of ischaemic heart disease, cerebrovascular disease, and other cardiovascular diseases, however, differs strongly by underlying cause. Many patients with cardiovascular disease as the underlying cause have a different manifestation of cardiovascular disease as a competing cause, and the prevalence of manifestations of cardiovascular disease is very high too among patients dying from respiratory diseases or from other diseases. There is also substantial variation in prevalence of all other diseases - a heterogeneous category within which the two most frequent competing causes are diabetes mellitus and senile dementia.

Table 2 presents estimates of gains in life expectancy after elimination of the four main groups of underlying causes of death, according to two different calculation methods. The conventional calculation method suggests that elimination of neoplasms as an underlying cause would result in 3.83 life years to be gained among men, and 3.38 life years to be gained among women. Elimination of cardiovascular diseases results in a larger gain in life expectancy: 4.93 years among men and 4.52 years among women. Elimination of respiratory diseases and other diseases results in smaller gains in life expectancy.

If you take into account the higher prevalence of competing causes among persons dying from other underlying causes than neoplasms, the numbers of life years gained by elimination of these other causes become smaller. Note that, because we cannot compare the prevalence of conditions identified as competing causes between those who have died and the total population, we can only adjust the relative differences in life years gained between underlying causes, not the absolute estimates of life years gained. This has been achieved by designating neoplasms as the reference category among the underlying causes of death, and by subsequently adjusting the estimates for the other underlying causes. Elimination of cardiovascular diseases now results in a gain in life expectancy, which, both among men and among women, is very close to the estimate for neoplasms. According to the conventional calculations, gains in life expectancy after elimination of cardiovascular diseases exceed those of

Table 2 Estimates of gains in life expectancy after elimination of four groups of underlying causes of death, with and without taking into account competing causes. The Netherlands, 1990

\begin{tabular}{lll}
\hline & \multicolumn{2}{c}{ Gain (in years) } \\
\cline { 2 - 3 } $\begin{array}{l}\text { Underlying cause of } \\
\text { death }\end{array}$ & $\begin{array}{l}\text { Conventional } \\
\text { calculation }\end{array}$ & $\begin{array}{l}\text { Revised calculation, } \\
\text { taking into account: } \\
\text { competing causes }\end{array}$ \\
\hline & Men & \\
Neoplasms & 3.83 & 3.83 \\
Cardiovascular diseases & 4.93 & 4.02 \\
Respiratory diseases & 0.82 & 0.41 \\
Other diseases & 2.51 & 1.91 \\
& Women & 3.38 \\
Neoplasms & 3.38 & 3.37 \\
Cardiovascular diseases & 4.52 & 0.28 \\
Respiratory diseases & 0.57 & 1.92 \\
Other diseases & 2.76 & \\
\hline
\end{tabular}

^Reference category
KEY POINTS

- Because of the problem of "competing causes of death", conventional estimates of the number of life years to be gained by elimination of specific causes of death are too generous.

- Among persons dying from neoplasms, the prevalence of diseases that could act as competing causes of death, is higher than among persons dying from cardiovascular diseases.

- When "competing causes" are ignored, the relative impact of eliminating cardiovascular diseases, as compared with eliminating neoplasms, will be overestimated.

neoplasms by more than one year, or approximately $30 \%$. According to the revised calculations, taking into account competing causes of death, the advantage for cardiovascular diseases dwindles to $5 \%$ among men and $0 \%$ among women, suggesting that both underlying cause of death groups are equally important in terms of potential gains in life expectancy. Respiratory diseases and other diseases fall even farther behind neoplasms than in the conventional calculations.

\section{Discussion}

Our study represents an attempt to assess a widely suspected bias in conventional estimates of life years to be gained by elimination of a cause of death. On the basis of death certificate information, the only conceivable data source covering sufficient numbers of deaths, we estimated differences between four main groups of underlying causes of death in the prevalence of competing causes of death. We then used these estimates to adjust conventional cause elimination life table calculations, and found that differences between underlying causes of death in life years to be gained are seriously biased if differences in the prevalence of competing causes are not taken into account. Specifically, the relative impacts of eliminating cardiovascular diseases and respiratory diseases, as compared with eliminating neoplasms, are overestimated.

Our study has several limitations:

Firstly, we relied on the contents of death certificates, and this is not a perfect source of information on underlying causes, let alone competing causes. ${ }^{20}{ }^{21}$ The death certificate has never been designed to elicit information on competing causes of death. Actually, the WHO guidelines on completing the death certificate discourage the notification of competing causes of death. In part I, all conditions listed should be a consequence of the underlying cause, while in part II only conditions that contributed to death should be mentioned. In practice, however, physicians rather undiscriminatively list all conditions that they think may be of relevance. Also, even within the limitations of the format of the certificate, completion is frequently suboptimal because of lack of relevant diagnostic information or simply because of lack of motivation on the part of the 
physician. Finally, the only information on competing causes that may reach the death certificate relates to conditions that are present at the moment the patient dies from his or her underlying cause. Still, we see no reason to assume that these imperfections are different between underlying causes of death and therefore, data on other conditions mentioned on the death certificate can at least be used to assess the bias present in comparisons between underlying causes of death of life years to be gained by elimination.

Secondly, an important assumption on which our (revised) calculations are based, is that the increased death rate among those with a competing cause present at the time they are saved from dying from a specific underlying cause of death, remains increased during the rest of their lives. Because for a saved person the number of life years gained can be substantial (the average ranges between 8 and 15 years for the underlying cause of death groups in table 2), this is unlikely to be realistic. It is more likely that the mortality risks among those with a competing cause present will gradually converge towards the values for those who are saved from the same underlying cause without a competing cause being present. The rate at which this happens can only be a matter of speculation, and we have restricted ourselves to a sensitivity analysis by assuming an arbitrary but reasonably strong convergence rate. This convergence rate, which equals a $10 \%$ reduction of excess mortality per additional year of life after the moment of being saved, implies a $65 \%$ reduction over a 10 year period. For example, the convergence scenario assumes that the effect of a higher prevalence of competing causes among persons dying from cardiovascular diseases will gradually diminish over time, to $90 \%$ of the original effect after one year, $35 \%$ after 10 years, etc. Naturally, this reduces the effect of taking into account competing causes of death (table 3, first column). The numbers of life years gained by elimination of cardiovascular diseases, respiratory diseases and other diseases increase relative to that of neoplasms, and elimination of cardiovascular diseases again becomes slightly more important than elimination of neoplasms.

Thirdly, the co-occurrence of two conditions at the time of dying not only may signify the presence of an underlying and a competing cause of death, but may also imply the presence of an underlying and a "contributing" cause of death. Consider a person dying from ischaemic heart disease as an underlying cause of death, with chronic obstructive lung disease being present as a comorbid condition and competing cause. Elimination of ischaemic heart disease will in this case result in a smaller gain in life expectancy than is suggested by conventional cause elimination life table calculations. Now consider another person, this one dying from chronic obstructive lung disease as an underlying cause but with ischaemic heart disease present as a comorbid condition and competing cause. What is the effect of elimination of ischaemic heart disease on the life chances of this person? If ischaemic heart disease has
Table 3 Estimates of gains in life expectancy after elimination of four groups of underlying causes of death, taking into account competing causes plus convergence or contributing causes

\begin{tabular}{lll}
\hline & $\begin{array}{l}\text { Gain (in years) } \\
\text { Revised calculation taking into } \\
\text { account competing causes plus: }\end{array}$ \\
\cline { 2 - 3 } $\begin{array}{l}\text { Underlying cause of } \\
\text { death }\end{array}$ & Convergencet & $\begin{array}{l}\text { Contributing } \\
\text { causes }\end{array}$ \\
\hline Neoplasms & Men & \\
Cardiovascular diseases & 3.83 & 3.90 \\
Respiratory diseases & 4.34 & 4.55 \\
Other diseases & 0.49 & 0.43 \\
Neoplasms & 2.15 & 2.16 \\
Cardiovascular diseases & 3.38 & 3.42 \\
Respiratory diseases & 0.33 & 3.85 \\
Other diseases & 2.16 & 0.29 \\
\end{tabular}

$\star$ Reference category. $\dagger 10 \%$ reduction of excess mortality per additional year of life after the moment of being saved.

acted as a "contributing" cause of death, increasing the risks of dying in a person already suffering from chronic obstructive lung disease, it is likely that elimination of ischaemic heart disease will result in a certain increase in life expectancy of this second person. The size of this effect is, again, a matter of speculation and thus a source of uncertainty. We have therefore tried to estimate the maximum size this effect could have, by assuming that elimination of a particular underlying cause would also save all those persons who died from another underlying cause but in whom that condition was present too (for example, elimination of ischaemic heart disease would also save persons dying from chronic obstructive lung disease with ischaemic heart disease as a comorbid condition). The results are presented in the second column of table 3. This modification of the calculation procedure increases the estimates of the number of life years to be gained by eliminating neoplasms and respiratory diseases by only 0.01 to 0.07 years. On the other hand, the effects on the estimates for cardiovascular diseases and other diseases are more substantial: these are relatively frequent comorbid conditions, acting both as competing and as contributing causes. In this fourth series of calculations, the effect of elimination of cardiovascular diseases again becomes more important than that of elimination of neoplasms, but it should be recognised that these calculations almost certainly overestimate the importance of cardiovascular diseases as a contributing cause. The reason is that not all those who die with cardiovascular diseases as a comorbid condition will be saved by elimination of this cause of death.

Although both modifications of the calculation procedure as presented in table 3 modify the impact, relative to neoplasms, of eliminating cardiovascular diseases, respiratory diseases and other diseases, the overall conclusion still holds that conventional calculations seriously overestimate the relative impacts on life expectancy of these three groups of conditions.

What are the implications of these findings?

In terms of impact on life expectancy, cardiovascular diseases have for decades been the main cause of death in the Netherlands as well 
as elsewhere in Western Europe and North America. ${ }^{22-24}$ With the decline of cardiovascular disease mortality, which started around 1970 in most countries, the relative importance of neoplasms has increased. This is all well known, but the radical nature of these changes has not yet been fully appreciated, because the conventional approach to estimate the impact of causes of death on life expectancy overestimates the relative importance of cardiovascular diseases. Our revised estimates, which take into account differences between causes of death in prevalence of competing causes, show that neoplasms and cardiovascular diseases have become (nearly) equally important in the Netherlands in the early 1990s. Although other countries in Western Europe and North America may be in different phases of epidemiological development, it is likely that the higher prevalence of competing causes among persons dying from cardiovascular disease than among those dying from neoplasms is a generalised phenomenon. Sooner than most observers realise, further increases in life expectancy will be as dependent upon progress in the prevention and treatment of cancer, as they are upon further declines in cardiovascular mortality.

Our results also illustrate an important lesson for intervention programmes. The number of life years to be gained by intervention is likely to be reduced because of the presence of competing causes of death. However, these competing causes will only reduce the number of life years to be gained, if the intervention does not eliminate the competing causes with the underlying cause. Because associations between competing and underlying causes depend on common risk factors, the effect of competing causes of death is likely to be smaller with interventions targeted at risk factors than with interventions targeted at more advanced steps in the disease process, for example, medical treatment of symptomatic disease. Primary prevention therefore remains the intervention of first choice, especially in the case of cardiovascular disease.

Funding: this study was financially supported by the Dutch Ministry of Public Health, Welfare and Sports.

\section{Conflicts of interest: none.}

1 Chiang CL. Life table and mortality analysis. Geneva: World Health Organization, 1978.

2 Preston SH, Keyfitz N, Schoen R. Causes of death: life tables for national populations. New York: Seminar Press, 1972

3 US Der national populations. New York: Seminar Press, 1972 . US Department of Health and Human Services. United States life table eliminating certain causes of death, 1979-1981.
DHSS Publ No. (PHS) 88-1150-2. Washington: Public DHSS Publ No. (PHS)

4 Tsai SP, Lee ES, Hardy RJ. The effect of a reduction in leading causes of death: potential gains in life expectancy. Am $\mathcal{F}$ Public Health 1978;68:966-71.

5 Keyfitz N. What difference would it make if cancer were eradicated? An examination of the Taeuber paradox. Demography 1977;14:411-18.

6 Chiang CL. Competing risks in mortality analysis. Annu Rev Publ Health 1991;12:281-307.

7 Cornfield J. The estimation of the probability of developing a disease in the presence of competing risks. Am f Public Health 1957;47:601-7.

8 Prentice RL, Kalbfleisch JD, Peterson AV, et al. The analysis of failure times in the presence of competing risks. Biometrics 1978;34:541-54.

9 Manton KG, Poss SS. Effects of dependency among causes of death for cause elimination life table strategies. Demograof death for cause elin

10 Manton K, Stallard E. Recent trends in mortality analyses. New York: Academic Press, 1986.

11 Israel RA, Rosenberg HM, Curtin LR. Analytical potential for multiple cause-of-death data. Am $\mathcal{F}$ Epidemiol 1986;124 161-79.

12 Puffer RR. New approaches for epidemiologic studies of mortality statistics. Bull Pan Am Health Organ 1989;23: $365-83$.

13 Steenland K, Nowlin S, Ryan B, et al. Use of multiple-cause mortality data in epidemiologic analysis: US rate and proportion files developed by the National Institute for Occupational Safety and Health and the National Cancer Institute. Am ₹ Epidemiol 1992;136:855-62.

14 Buich D. Automatic coding of causes of death. Popul Trends 1993;73:36-8

15 Mackenbach JP, Kunst AE, Lautenbach $\mathrm{H}$, et al. Competing causes of death: an analysis using Multiple-Cause-of-Death Data from The Netherlands. Am 7 Epidemiol 1995;5:46675 .

16 Flaten TP. Mortality from dementia in Norway, 1969-83. $f$ Epidemiol Community Health 1989;43:285-9.

17 Wong O. A competing-risk model based on the life table procedure in epidemiological studies. Int f Epidemiol 1977; 6:153-9.

18 World Health Organization. Manual of the International Statistical Classification of Diseases, Injuries and Causes of Death. 9th revision. Geneva: World Health Organization, 1977.

19 Mackenbach JP, Kunst AE, Lautenbach $\mathrm{H}$, et al. Competing causes of death: a death certificate study. 7 Clin Epidemiol 1997;50:1069-77.

20 Bild DE, Stevenson JM. Frequency of recording of diabetes on US death certificates: analysis of the 1986 national mortality followback survey. $\mathcal{F}$ Clin Epidemiol 1992;45:275-81.

21 Goldacre MJ. Cause-specific mortality: understanding uncertain tips of the disease iceberg. $\mathcal{F}$ Epidemiol Community Health 1993;47:491-6.

22 Uemura $\mathrm{K}$, Pisa $\mathrm{Z}$. Trends in cardiovascular disease mortality in industrialized countries since 1950. World mortality in industrialized co
Health Stat $Q 1988 ; 41: 155-78$.

23 Thom TJ, Epstein FH, Feldman JJ, et al. Trends in total mortality and mortality from heart disease in 26 countries from 1950-1978. Int f Epidemiol 1985;14:510-20.

24 Beaglehole R. International trends in coronary heart disease mortality, morbidity and risk factors. Epidemiol Rev 1990;12:1-15. 